\title{
Upregulation of miR-223 in the rat liver inhibits proliferation of hepatocytes under simulated microgravity
}

\author{
Yongjie Chen ${ }^{1,2,4}$, Ji Xu ${ }^{2,3,4}$, Chao Yang ${ }^{2}$, Hongyu Zhang ${ }^{2}$, Feng $\mathrm{Wu}^{2}$, Jian $\mathrm{Chen}^{2}$, Kai $\mathrm{Li}^{2}$, Hailong Wang ${ }^{2}$, \\ $\mathrm{Yu} \mathrm{Li}{ }^{1,3}$, Yinghui $\mathrm{Li}^{2,3}$ and Zhongquan $\mathrm{Dai}^{2}$
}

Long-term spaceflight affects numerous organ systems in the body, including metabolic dysfunction. Recently, ample evidence has demonstrated that the liver is a vulnerable organ during spaceflight. However, the changes in hepatocyte proliferation and cell cycle control under microgravity remain largely unexplored. In the present study, we first confirmed that the serum levels of aspartate aminotransferase, alanine aminotransferase and alkaline phosphatase, biochemical markers of liver function, were altered in rats under tail suspension (TS) conditions to simulate microgravity, as shown in previous reports. Next, we demonstrated that the cell proliferation activity, determined by Ki67, PCNA and PH3, was significantly decreased at the different TS time points (TS for 14, 28 and 42 days) compared with that in the control group. Consistently, the positive cell cycle regulators Ccna2, Ccnd1, Cdk1, Cdk2 and cyclin D3 were also significantly decreased in the TS groups as shown by quantitative real-time PCR and western blotting analysis. Subsequent analysis revealed that the aberrant hepatocyte proliferation inhibition under simulated microgravity was associated with the upregulation of miR-223 in the liver. We further found that miR-223 inhibited the proliferation of Hepa1-6 cells and identified CDK2 and CUL1 as its direct targets. In addition, the decreased expression of CDK2 and CUL1 was negatively correlated with the level of p27 in vitro and in vivo, which may have been responsible for retarding hepatocyte proliferation. Collectively, these data indicate that upregulation of miR-223 was associated with the inhibition of liver cell growth and reveal the role of miR-223 in rat hepatocyte proliferation disorders and the pathophysiological process under simulated microgravity.

Experimental \& Molecular Medicine (2017) 49, e348; doi:10.1038/emm.2017.80; published online 23 June 2017

\section{INTRODUCTION}

Spaceflight causes some significant physiological and pathological changes in various organ systems of astronauts. ${ }^{1}$ The main physiological effects of spaceflight in the body are bone loss, muscle atrophy, metabolic disorder and cardiovascular problems. ${ }^{2}$ The liver plays important roles in maintaining metabolic homeostasis. Carbohydrates, lipids and enzymes have been reported to be changed under microgravity. ${ }^{3-6}$ Several studies have demonstrated that glycogen, alanine aminotransferase (ALT) and aspartate aminotransferase (AST) of the liver were significantly impacted by spaceflight in humans and rats and by tail suspension (TS) in rats, ${ }^{7-9}$ which suggested that microgravity would lead to liver function derangement. In addition to these alterations of biochemical indexes, simulated microgravity (SMG) induced mild portal endotoxemia, which may contribute to hepatic injury and trigger hepatic apoptosis. ${ }^{8,9}$ These results support the view that the liver is a vulnerable organ during spaceflight. We know that hepatocyte proliferation is associated with various types of liver injuries. ${ }^{10,11}$ However, the changes and mechanism of hepatocyte proliferation under microgravity have not been elucidated.

The liver is an amazing organ that has the extraordinary capacity to regenerate from various types of injuries and maintain some vital functions. ${ }^{12,13}$ The liver is capable of restoring $70 \%$

\footnotetext{
${ }^{1}$ School of Computer Science and Technology, Harbin Institute of Technology Shenzhen Graduate School, Shenzhen, Guangdong, China; ${ }^{2}$ State Key Laboratory of Space Medicine Fundamentals and Application, China Astronaut Research and Training Center, Beijing, China and ${ }^{3}$ School of Life Science and Technology, Harbin Institute of Technology, Harbin, China

${ }^{4}$ These authors contributed equally to this work.

Correspondence: Professor Yu Li, School of Computer Science and Technology, Harbin Institute of Technology Shenzhen Graduate School, Shenzhen, Guangdong 518055, China.

E-mail: liyugene@hit.edu.cn

or Professor YH Li or Dr Z Dai, State Key Laboratory of Space Medicine Fundamentals and Application, China Astronaut Research and Training Center, No. 26, Beiqing Road, Haidian District, Beijing 100094, China.

E-mail: yinghuidd@vip.sian.com or daizhq77@163.com

Received 28 August 2016; revised 10 January 2017; accepted 17 January 2017
} 
tissue loss within a few weeks, which depends on the activation of dormant stem cells, transdifferentiation, metaplasia or compensatory proliferation of quiescent hepatocytes. ${ }^{11}$ Liver regeneration is a highly orchestrated process controlled by coordinated signaling events, including inflammatory factors, growth factors, miRNAs and metabolic networks that link liver function to cell growth and proliferation. ${ }^{10}$ During acute injury, inflammatory factor (TNF $\alpha$, IL-6 and so on) signaling directs an immediateearly gene expression program that induces hepatocytes to re-enter the cell cycle, proliferate and replenish the lost tissue. ${ }^{14-16}$ Increasing evidence has suggested that cell proliferation, apoptosis, tissue remodeling and tumorigenesis are tightly regulated by microRNAs (miRNAs). ${ }^{17-21}$ Altered expression of miRNAs has been implicated in a variety of liver diseases, although the underlying mechanisms of the influence of miRNAs on liver disease remain largely unknown. ${ }^{20}$ MiRNAs have been demonstrated to be able to regulate hepatocyte proliferation during liver regeneration in a rodent partial hepatectomy model. For example, miRNA-21, miR-382 and miRNA-221 accelerate hepatocyte proliferation after partial hepatectomy by targeting cyclin D1, PTEN-Akt axis and Arnt, respectively. ${ }^{17,22,23}$ Other results have shown that downregulation of miR-127 and miR-26a promotes mouse hepatocyte proliferation during liver regeneration by targeting CCNE2 and Bcl6, Setd8 and CCND2, respectively. $^{24,25}$ MiR-9a, miR-223, miR-27a, miR-133a, miR-145, miR-150 and miR-153 have been shown to be involved in liver cell proliferation and cell cycle control. ${ }^{26-30}$ Previous results have demonstrated that miR-223 is widely expressed in multiple cell types and tissues, including myeloid cells, ${ }^{31}$ hepatocytes, ${ }^{32,33}$ cardiomyocytes, ${ }^{34}$ the ovary ${ }^{35}$ and prostate, ${ }^{36}$ and play important roles in inflammation, type 2 diabetes, lipid and cholesterol metabolism, bone metabolism and cell proliferation. Multiple cell cycle regulators such as E2F1, Fbxw7/Cdc4, IGF-1R, Cdk2 and TOX have been indicated to be regulated by miR-223. ${ }^{37-40}$ In view of these data, miR-223 could regulate the cell cycle control in hepatocytes. Recently, changes in miRNA expression profiles have been shown in mammalian cells and organs under SMG, ${ }^{41-44}$ but the involvement of miRNAs in the regulation of liver function and hepatocyte proliferation has rarely been reported.

Here we demonstrated that the enzyme activity of ALT, AST and alkaline phosphatase (ALP) was considered abnormal and hepatocyte proliferation was inhibited in rats under SMG. The further investigations showed that the elevation of miR-223 contributed to the retarded hepatocyte proliferation, at least in part, by targeting CDK2 and CUL1. In addition, the decreased level of CDK2 and CUL1 was related to the upregulation of p27 in vitro and in vivo, which may be responsible for retarding hepatocyte proliferation. Our findings provide a new role for miR-223 in the regulation of the physiological and pathological function of the liver.

\section{MATERIALS AND METHODS}

\section{Animals}

All animal protocols were performed in accordance with the standard ethical guidelines and were approved by the Institutional Review
Board of China Astronaut Research and Training Center. Sprague Dawley rats were purchased from the Animal Center of the Academy of Military Medical Sciences (Beijing, China). Forty male rats aged 7 weeks were allowed to acclimatize to their new surrounding for 1 week as singletons, and had free access to water and standard chow under a $12 \mathrm{~h} / 12 \mathrm{~h}$ light/dark cycle.

\section{Tail suspension rat model}

The rats were randomly assigned into the control group $(n=10)$ and the TS groups $(n=30)$. The experimental time line is shown in Supplementary Figure S1. Rats in the TS groups were tail-suspended as in the following description for 6, 4 or 2 weeks (TS-42, TS-28 and TS-14 group, respectively; 10 rats in each group). The TS procedure was performed as previously described ${ }^{45}$ with minor modifications. Briefly, the rats were suspended by their tails using a strip of adhesive surgical tape attached to a chain hanging from a beam. The rats were posed at a $30^{\circ}$ angle to the floor with only the forelimbs touching the floor and were allowed to move freely to have food and water. The control rats were singly housed in the same cage without TS.

\section{Tissue collection}

At the end of the animal experiment, all rats were anesthetized with $1 \%$ sodium pentobarbital $\left(45 \mathrm{mg} \mathrm{kg}^{-1}\right)$. The blood was collected by cardiac puncture. The whole blood was separated into serum and cellular fractions by centrifugation at $2000 \mathrm{~g}$ for $10 \mathrm{~min}$ at $4^{\circ} \mathrm{C}$. The supernatant serum was stored at $-80^{\circ} \mathrm{C}$ until analysis. The liver was fixed with $4 \%$ paraformaldehyde for immunostaining or frozen in liquid nitrogen for gene expression analysis.

Serum samples were performed to detect the level of ALT, AST and ALP liver enzymes for liver function by biochemical analysis (Beijing North Institute of Biological Technology, China).

\section{Cell culture}

Hepa1-6 cells were cultured in Dulbecco's Modified Eagle Medium (HyClone, Logan, UT, USA) supplemented with 10\% fetal bovine serum, streptomycin $\left(100 \mu \mathrm{g} \mathrm{ml}^{-1}\right)$ and penicillin $\left(100 \mathrm{U} \mathrm{ml}^{-1}\right)$ at $37^{\circ} \mathrm{C}$ with $5 \% \mathrm{CO}_{2}$. Hepal-6 cells were plated in 12-well plates with $1 \times 10^{5}$ cells per well for $24 \mathrm{~h}$. Then, $100 \mathrm{~nm}$ of miR-223 mimic or negative control miRNA (Genepharma, Suzhou, China) was transiently transfected using Lipofectamine 3000 reagent (Invitrogen, Carlsbad, CA, USA) for $48 \mathrm{~h}$. The cells were then lysed by RIPA buffer for western blotting.

\section{Cell proliferation assay}

Cell proliferation assays were performed using the MTS reagent (Promega, Madison, WI, USA) according to the manufacturer's instructions. In brief, Hepa1-6 cells were seeded on 96-well plates with $8 \times 10^{3}$ cells per well and, once $70 \%$ confluent, subjected to transfection with miR-223 mimic or negative control (Genepharma) using Lipofectamine 3000. After 24, 48 or $72 \mathrm{~h}$ of transfection, MTS was added at $20 \mu \mathrm{l}$ per well for $2 \mathrm{~h}$. Absorbance of the supernatant was measured at $490 \mathrm{~nm}$ using a microplate reader ( $\mu$-Quant; Bio-Tek Instruments, Winooski, VT, USA). All experiments were repeated three times.

\section{Quantitative real-time PCR}

Total RNA was isolated from rat liver by using standard TRIzol-based protocols according to the manufacturer's instructions. Here, $1 \mu \mathrm{g}$ total RNA was used to synthesize complementary DNA by using the PrimeScript RT reagent Kit (Takara, Dalian, China; code no.: 

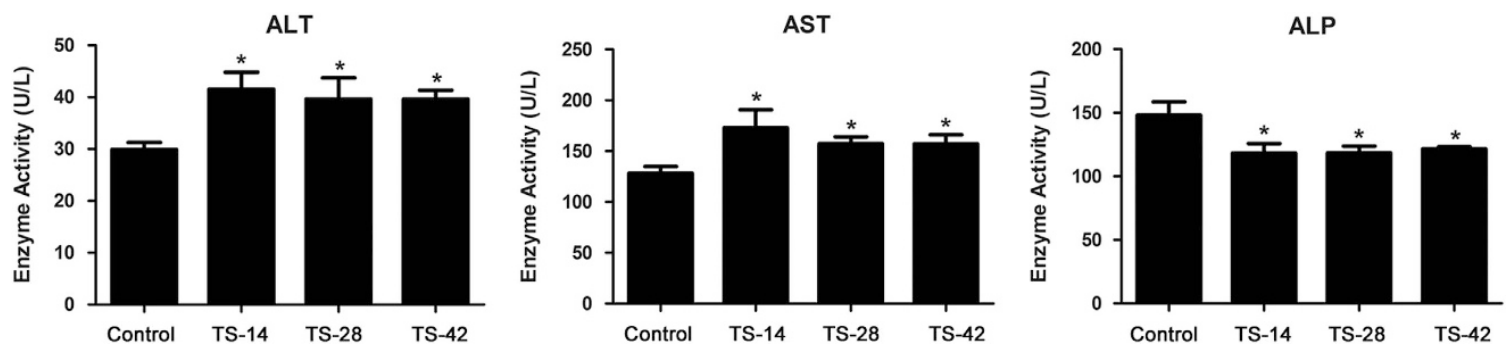

Figure 1 Liver function was impaired under SMG. SMG significantly increased the serum levels of ALT and AST but decreased the serum level of ALP after TS for 14, 28 and 42 days (TS-14, TS-28 and TS-42) compared with that in the control group. $n=10$. ${ }^{*} P<0.05$ compared to Control. ALT, alanine aminotransferase; AST, aspartate aminotransferase; SMG, simulated microgravity.

RR047A). For the miRNA assay, the miRNAs were reverse transcribed with the Mir-X miRNA First-Strand Synthesis Kit (Takara; code no.: 638313). Expression levels of different cell cycle genes and miRNAs were detected by a SYBR Premix Ex Taq II (Tli RNaseH Plus) Kit (Takara; code no.: RR820A) using the ROCHE LightCycler 96 system (Roche, Basel, Switzerland). The rat genes of $\beta$-actin or U6 were used as internal reference genes for mRNA and miRNA, respectively. All of the cell cycle genes and miRNA primers for reverse transcriptionquantitative polymerase chain reaction (RT-qPCR) are listed in Supplementary Tables S1 and S2.

\section{Immunostaining}

All of the fixed samples were dehydrated in a graded alcohol series. Tissue samples were eventually embedded in paraffin before being sectioned. The rehydrated sections were employed for immunostaining. Primary antibodies specific to Ki67 (Epitomics, Burlingame, CA, USA), PH3 (Cell Signaling Technology, USA) and PCNA (Cell Signaling Technology, Beverly, MA, USA) were used. Sections incubated without primary antibodies were used as negative controls. High-temperature heating was used for antigen retrieval. A HistostainSP Kit (Zhongshan Golden Bridge Biotechnology, Beijing, China) was applied to visualize the antigen. For immunofluorescence, liver sections $(8 \mu \mathrm{m})$ were fixed in $4 \%$ paraformaldehyde solution. Primary antibodies specific to p27 (ABclonal Technology, Wuhan, China) were used, and the sections were stained with Cy3-labeled secondary antibodies (Jackson ImmunoResearch Laboratories, West Grove, PA, USA) before being counterstained with 4',6-diamidino-2-phenylindole. Immunofluorescence images were captured via a Leica TCS SP5 confocal scanning laser microscope (Leica, Solms, Germany).

\section{Western blotting}

Liver and whole-cell lysates were prepared using RIPA buffer (50 mM Tris-HCl (pH 7.4), $150 \mathrm{~mm} \mathrm{NaCl,} \mathrm{1 \%} \mathrm{NP-40,} 1$ mm EDTA and $0.1 \%$ sodium dodecyl sulfate) containing protease inhibitor cocktail (Roche) and then each sample was kept on ice for $30 \mathrm{~min}$ before it was centrifuged at 13000 r.p.m. for $30 \mathrm{~min}$ at $4{ }^{\circ} \mathrm{C}$. The protein concentration of the supernatant was determined with a bicinchoninic acid protein assay kit according to the manufacturer's instructions (Thermo Fisher Scientific, Waltham, MA, USA). Proteins were subjected to $10 \%$ polyacrylamide gel electrophoresis and then transferred onto a polyvinylidene difluoride membrane (Millipore, Bedford, MA, USA). Membranes were blocked with 5\% skim milk in Tris-buffered saline with $0.1 \%$ Tween 20 for $1 \mathrm{~h}$ and then incubated overnight with a primary antibody diluted in 5\% bovine serum albumin at $4{ }^{\circ} \mathrm{C}$. Specific antibodies to p27, CDK2, CUL1 (ABclonal Technology), PCNA (Santa Cruz Biotechnology, Santa Cruz, CA, USA), cyclin D1 (Invitrogen), cyclin D3 and GAPDH
(Cell Signaling Technology) were used. GAPDH served as a loading control. The membranes were incubated in appropriate secondary antibodies conjugated to horseradish peroxidase (Cell Signaling Technology) for $2 \mathrm{~h}$. The signals were detected using enhanced luminescence (Bio-Rad, Hercules, CA, USA) after washing three times. The intensity of the protein bands was quantified by using ImageJ software (National Institutes of Health, Windows version, Bethesda, MD, USA).

Recombinant plasmid construction and dual luciferase assay The 3'-UTR fragments of CDK2 and CUL1 containing the predicted miR-223-binding sites were amplified by PCR using the primers (Supplementary Table S3) and cloned into the pGL3-Promoter plasmid (Promega) downstream of the promote, with the resulting fragments named pGL3-WT-CDK2 and pGL3-WT-CUL1. Site-directed mutant vectors (base substitutions) were generated by using the KOD Plus Mutagenesis Kit (TOYOBO, Osaka, Japan) following the manufacturer's instructions and named pGL3-MUTCDK2 and pGL3-MUT-CUL1. For the luciferase reporter assays, Hepal-6 cells with 70\% confluence were grown in 48-well plates and transfected with $100 \mathrm{ng}$ of WT or mutant vectors (pGL3-MUT-CDK2, pGL3-MUT-CUL1, pGL3-MUT-CDK2 and pGL3-MUT-CUL1) and $100 \mathrm{~nm}$ miR-223 mimic (Genepharma) or negative control using Lipofectamine 3000. As an internal control, $10 \mathrm{ng}$ pRL-TK vector (Promega) was co-transfected for normalization of the transfection efficiency. Cells were lysed and the luciferase reporter activity was assayed $48 \mathrm{~h}$ after transfection using the dual luciferase assay kit (Promega) following the manufacturer's protocol.

\section{Statistical analysis}

Each experiment was repeated at least three times with independent samples. Statistical analysis was performed using a two-tailed Student's $t$-test by SPSS software (version 17.0; SPSS, Chicago, IL, USA). The data are expressed as the mean \pm s.e.m. $P$-value $<0.05$ was considered statistically significant $\left({ }^{\star} P<0.05,{ }^{* *} P<0.01\right)$.

\section{RESULTS}

\section{Liver function was impaired under SMG}

It has been firmly established that microgravity induces alteration of hepatic function and metabolism. ${ }^{7-9,46}$ In our study, we first detected the rat serum levels of AST, ALT and ALP, which are the classical biomarkers for liver function evaluation. As shown in Figure 1, the enzyme activity of AST and ALT were significantly raised in the TS-14, TS-28 and TS-42 groups. In contrast, SMG markedly decreased the enzyme activity of ALP in all three TS groups compared to that in the control groups. 
a



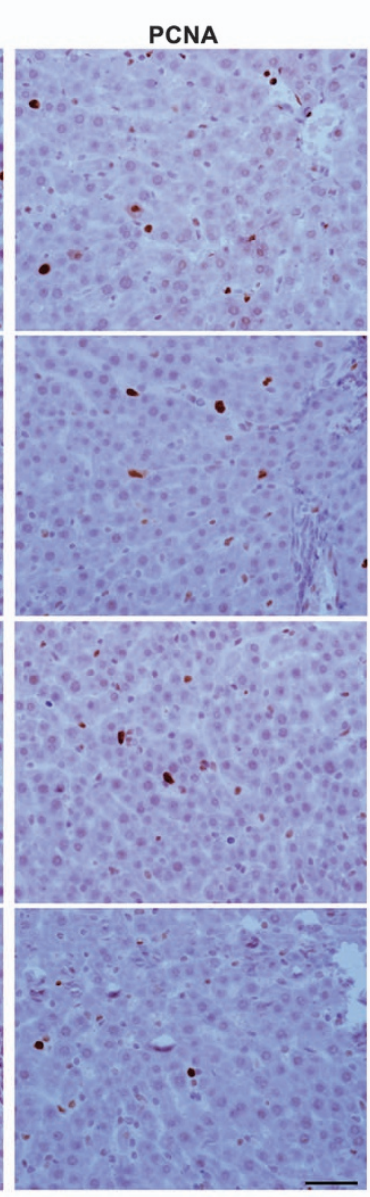

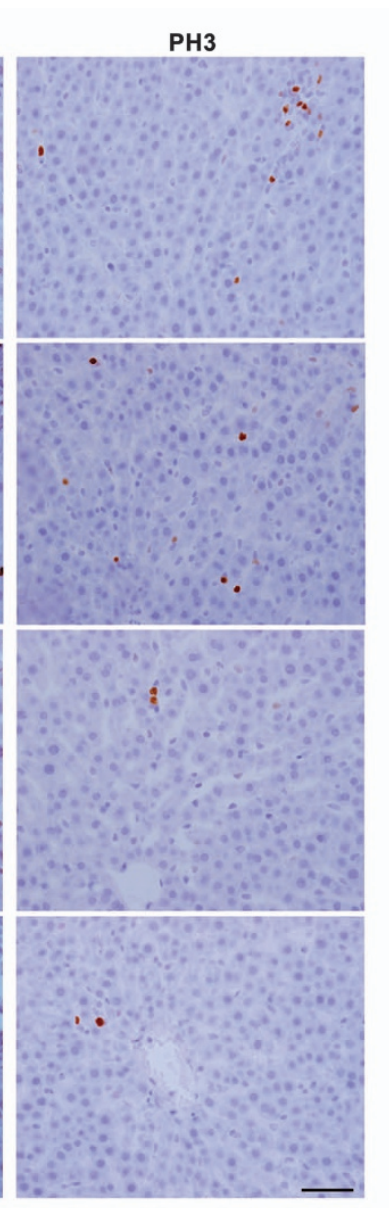

b
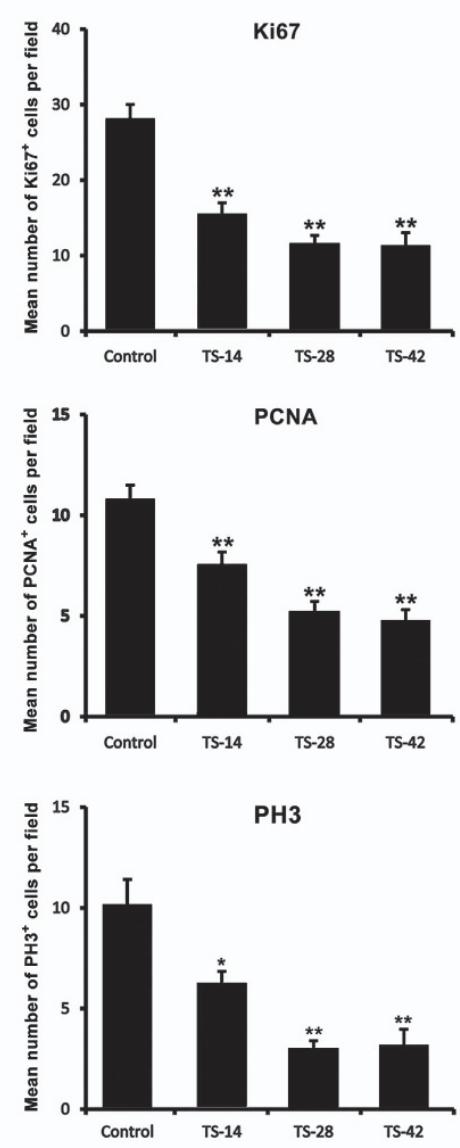

C


d
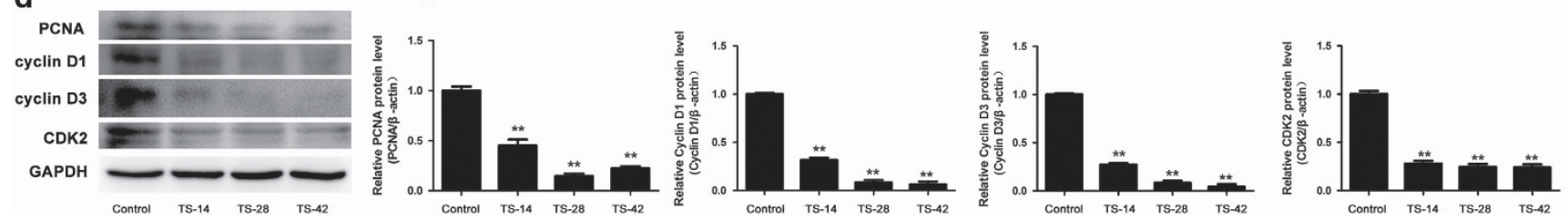

Figure 2 The effect of SMG on hepatocyte proliferation. (a) Immunostaining analysis of Ki67 (left panels), PCNA (center panels) and PH3 (right panels) in the rat liver of the control, TS-14, TS-28 and TS-42 groups. Scale bar, $50 \mu \mathrm{m}$. (b) Quantification of the Ki67-, PCNA- and PH3-positive cell number in the liver of the control, TS-14, TS-28 and TS-42 groups, $n=6$. (c) RT-qPCR analysis of gene expression related to cell cycle regulation such as CCNA2, CCND1, CDK1, CDK2 and FOXM1 in the rat liver of the control and TS groups. $n=6$. (d) Protein levels of PCNA, cyclin D1, cyclin D3 and CDK2 were analyzed by western blotting. $n=3$. The intensity of the protein bands was semiquantitatively analyzed using ImageJ software. ${ }^{*} P<0.05,{ }^{* *} P<0.01$ compared to Control. SMG, simulated microgravity; TS, tail suspension.

Ours and others' results demonstrated that SMG induced hepatic injury and impaired liver function.

\section{Hepatocyte proliferation was inhibited under SMG}

Hepatocyte proliferation is known to be essential for the maintenance of normal liver function under various types of injuries. Thus, we detected the hepatocyte proliferation status with Ki67, PCNA and PH3 immunostaining in experimental rats. As shown in Figure $2 a$ and $b$, the cell number of Ki67-positive hepatocytes was significantly decreased in the TS-14, TS-28 and TS-42 groups compared with that in the control group. The levels of PCNA, another marker of cell proliferation, also declined in the different TS groups as shown by the immunostaining and western blotting analysis (Figure $2 \mathrm{a}$ 
and d). We further detected the M-phase marker PH3 by using immunostaining. The results showed that SMG also diminished the cell number of PH3-positive hepatocytes in all of the investigated time points (Figure 2a and b), which was the same pattern observed with Ki67 and PCNA. These results demonstrated that fewer hepatocytes entered the cell cycle in the TS groups than the control group and the hepatocyte cell cycle was arrested by SMG.

Positive cell cycle regulators were downregulated under SMG To clarify the defects in cell cycle progression under SMG, we detected the expression of positive cell cycle regulators by using RT-qPCR and western blotting. As shown in Figure 2c, the mRNA expression of CCNA2 and CCND1 were inhibited in the TS-14, TS-28 and TS-42 groups compared with that in the control group. Downregulation of CDK1 and CDK2 was also found in all TS groups. Other cell cycle modulators such as CCNA1, CCNB1 and CKD4 did not show significant differences between the TS and control group (Supplementary Figure S2). We also found that expression of the proliferation-specific Forkhead Box M1 (FOXM1) gene was remarkably decreased in all TS groups by using RT-qPCR analysis (Figure 2c). In addition, compared with that of control, the protein levels of cyclin D1, cyclin D3 and CDK2 were markedly declined in the TS-14, TS-28 and TS-42 groups, as shown by the western blotting analysis (Figure 2d). These results revealed that the cell cycle control of hepatocytes was dysregulated under SMG.

\section{miR-223 was upregulated in the liver of TS rats}

Recently, miRNAs were found to play important roles under microgravity. ${ }^{41-44}$ Therefore, we screened a set of miRNAs that was demonstrated to participate in the regulation of cell proliferation using RT-qPCR analysis. We observed that miR-223 was elevated in the rat liver of the TS groups compared to that in the control group (Figure 3), while there were no notable changes in the expression of miR-9a, miR-27a, miR-133a, miR-145 and miR-153 detected at these TS time points (Figure 3). These results indicated that miR-223 might regulate hepatocyte proliferation under SMG.

miR-223 suppressed cell growth by targeting CDK2 and CUL1 To verify the biological role of miR-223 in hepatocyte proliferation, we transfected miR-223 mimic or negative control into Hepa1-6 cells. By using the MTS assay, we observed a significant decrease in the viability of Hepal-6 cells in the group transfected with miR-223 mimic compared with that in the negative control (Figure 4a). More importantly, the western blotting assay showed that the protein levels of PCNA and cyclin D1 were reduced in the miR-223 mimic treatment group compared with that in the control (Figure $4 \mathrm{~b}$ ). These results indicated that miR-223 could inhibit Hepal-6 cell growth in vitro.

To identify the direct targets of miR-223, we first predicted the targets using the online miRNA target databases (www.TargetScan.org) and found that Cdk2 and Culin1 (Cul1) may serve as potential targets of miR-223. Then, the $3^{\prime}-\mathrm{UTR}$ of Cdk2 and Cul1 containing the putative miR-223-binding sites were individually cloned into a luciferase expression plasmid (PGL3 promoter). We also generated their mutated vectors in which some nucleotides of the seed sequence were replaced as shown in Figure $5 a$ and $b$. Here, miR-223 mimic significantly reduced the luciferase activity of pGL3-WT-CDK2-3'-UTR and pGL3-WT-CUL1-3'-UTR, after normalizing to Renilla-luciferase transfection controls. However, miR-223 mimic failed to knockdown the luciferase activity of their mutated vectors (Figure $5 \mathrm{c}$ and $\mathrm{d}$ ).

To determine the effects of miR-223 on its targets, we transfected Hepal-6 cells with miR-223 mimic or negative control. As shown in Figure 5e, the protein levels of CDK2 and CUL1 were significantly reduced in miR-223 mimic-transfected cells, as indicated by western blotting analysis.

To verify the relationship between miR-223 and CUL1 in vivo, we detected the mRNA and protein level of CUL1 in the rat liver under SMG. As shown in Figure $5 \mathrm{f}$ and g, RT-qPCR and western blotting analysis showed that there were extremely lower levels of CUL1 mRNA and protein in the rat liver of the three TS groups. These results showed that the upregulated miR-223 was negatively associated with the expression of CUL1 and implied that miR-223 inhibited CUL1 expression in the liver under SMG. Thus, these results revealed that miR-223 targeted CDK2 (Figures $2 \mathrm{c}$ and $\mathrm{d}$, and $5 \mathrm{c}$ and $\mathrm{e}$,) and CUL1 (Figures $2 \mathrm{e}$, and $5 \mathrm{~d}, \mathrm{f}$ and $\mathrm{g}$ ) and suppressed their expression.

\section{SMG upregulated p27 in the rat liver}

CUL1, as an essential component of the SCF (SKP1-CUL1-F-box protein) E3 ubiquitin ligase complex, plays an important role during the ubiquitination-mediated degradation of $\mathrm{p} 27$, which is involved in cell cycle control by negatively regulating cyclin-CDK complexes. ${ }^{47-49}$ Previous reports also demonstrated that cyclin $\mathrm{E}-\mathrm{CDK} 2$ was a regulator of $\mathrm{p} 27^{47}$. Thus, we speculated that $\mathrm{p} 27$ could be regulated under SMG. The immunofluorescent staining and western blotting results showed that the p27-positive hepatocyte cell numbers were notably increased in the TS-14, TS-28 and TS-42 groups compared with that in control (Figure $6 \mathrm{a}$ and b), and the protein level of p27 was significantly upregulated in the three TS groups (Figure 6c). In vitro, miR-223 mimic significantly increased the protein level of p27 compared with that of the negative control (Figure 6d). These results suggested there should be a negative correlation between CUL1/CDK2 and p27 in vivo and in vitro.

\section{DISCUSSION}

The effects of microgravity on the liver began to be investigated after spaceflight missions in the 1980s, including its effects on carbohydrate and lipid metabolism and enzyme activity. ${ }^{3-6,50,51}$ Previous reports have shown that microgravity induces hepatic injury and apoptosis. ${ }^{89}$ It is well known that hepatocyte proliferation is associated with various types of injuries; however, less is known regarding the connection between liver function injury and hepatocyte cell cycle control under microgravity. Here we tried to uncover the answer to this question and explore the potential mechanism using a TS rat model to simulate microgravity. 



Figure 3 Expression changes of miRNA related to cell cycle control in the rat liver under SMG. RT-qPCR analysis of miRNA expression related to cell cycle control, including miR-223, miR-9a, miR-27a, miR-133a, miR-145, miR-150-5p, miR-153, miR-229a and miR-229b in the rat liver of the control, TS-14, TS-28 and TS-42 groups. $n=6,{ }^{*} P<0.05,{ }^{* *} P<0.01$ compared to Control. RT-qPCR, reverse transcription-quantitative polymerase chain reaction; SMG, simulated microgravity; TS, tail suspension.
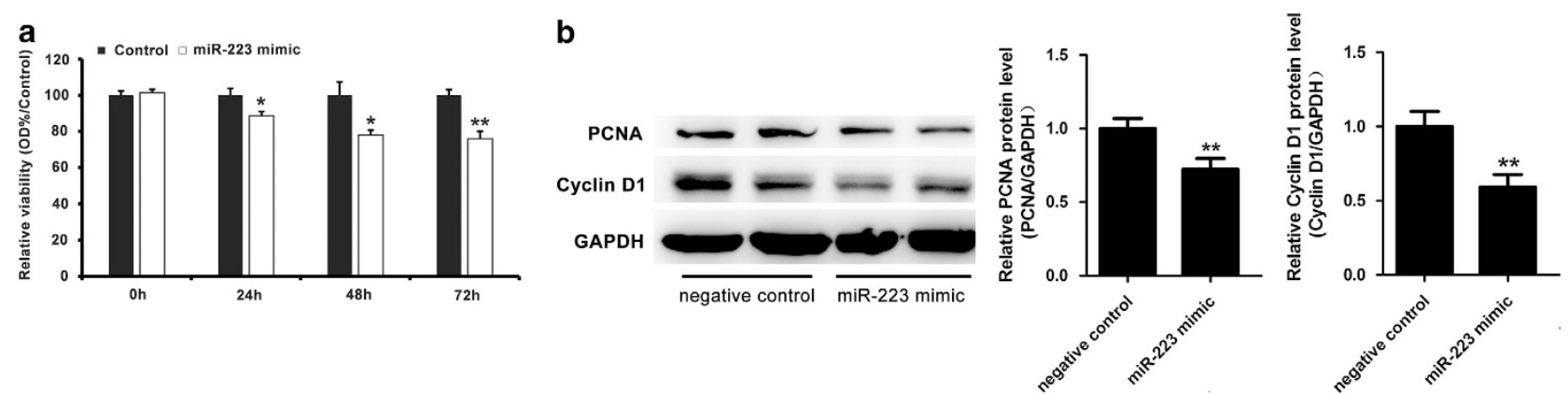

Figure 4 miR-223 suppressed Hepa1-6 cell proliferation in vitro. (a) The relative viability of Hepa1-6 cells was assessed with the MTS assay at different time points after transfection with miR-223 mimic or negative control. (b) Protein expression of the proliferation regulators PCNA and cyclin D1 in Hepa1-6 cells was examined $48 \mathrm{~h}$ after transfection with miR-233 mimic or the negative control by western blotting and semi-quantitatively analyzed using ImageJ software. ${ }^{*} P<0.05,{ }^{* *} P<0.01$ compared to Control.

In the present study, we first confirmed that the liver function was abnormal under SMG reflected by the enzyme activity of ALT, AST and ALP, which was consistent with previous reports and implied some degree of hepatic injury. Furthermore, using immunohistochemical staining, we found that the expression of proliferation markers Ki67, PCNA and PH3 was significantly decreased, which was coincident with the downregulation of positive cell cycle molecules (CCNA2, $C C N D 1, C D K 1$ and $C D K 2)$ in the TS rat liver. Therefore, our results indicated that microgravity potentially suppressed hepatocyte proliferation, which was not beneficial in the maintenance of liver homeostasis. Based on this, we should be concerned about the status of hepatocyte proliferation during long-term spaceflight in the future.

miRNAs are small noncoding RNAs and have been demonstrated to regulate a wide spectrum of biological processes. . $^{52,53}$ Recently, specific miRNAs have been shown to be altered under real microgravity or SMG conditions. ${ }^{41-44}$ Next, we detected several miRNAs that have been reported to mediate hepatocyte proliferation by RT-qPCR analysis and found that only miR-223 
a SV40 Lciferase b

\section{SV40}

\section{Lciferase}

3'UTR CUl1

WT 3'UTR Cul1 5' ...GCAGGACUGCUCAGGACUGACAC... (167-173) || || || || miR-223-3P $\quad 3^{\prime} \quad$ CCCCAUAAACUGUUUGACUGU

MUT 3'UTR Cul1 5' ...GCAGGACUGCUCAGGGGAAUUAC. (167-172)


g
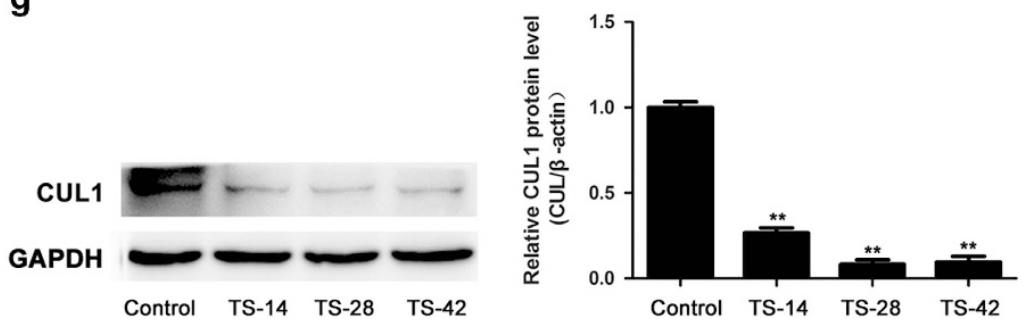

Figure 5 CDK2 and CUL1 were the direct targets of miR-223. Schematic representation of the luciferase constructs used for reporter assays. The miR-223 target sites within the 3'-UTR of CDK2 (a) and CUL1 (b) are shown as black boxes. The sequences below indicated the putative miR-223 target sites on the wild-type (WT) and mutated (MUT) constructs. The miR-223-binding sites and seed sequences are marked with bold italics. (c, d) Effects of miR-223 on the luciferase activity of the reporter gene in the WT or mutated vector in Hepa1-6 cells. $n=3$. (e) CDK2 and CUL1 protein expression in Hepa1-6 cells was examined after transfection with miR-233 mimic and negative control for $48 \mathrm{~h}$ and semi-quantitative evaluation using ImageJ software. mRNA (f) and protein (g) expression of CUL1 analyzed in the rat liver of the control and TS groups. $n=6,{ }^{*} P<0.05,{ }^{* *} P<0.01$ compared to Control. 
a
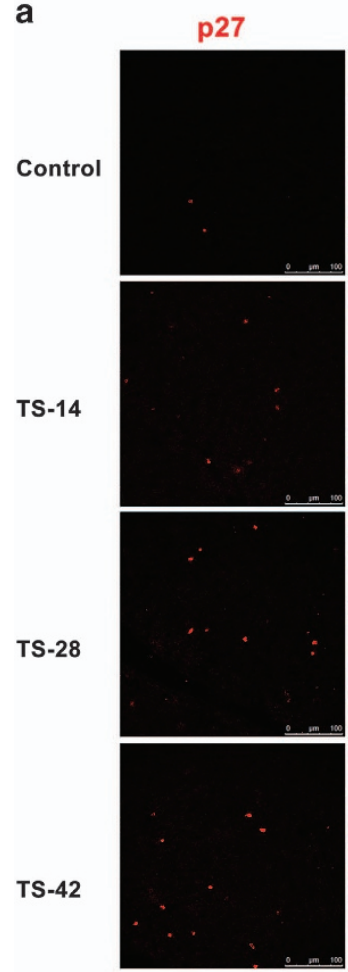

d
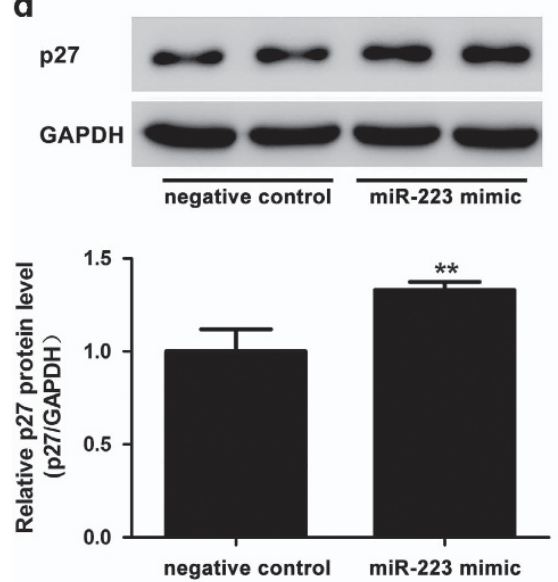

b

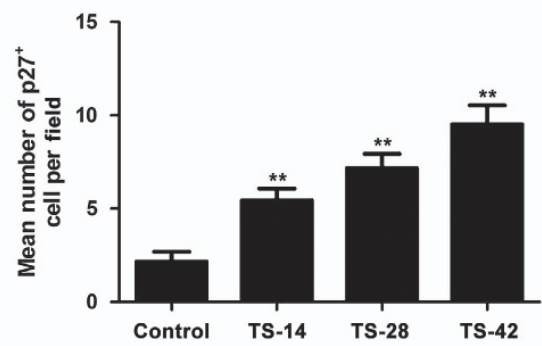

c
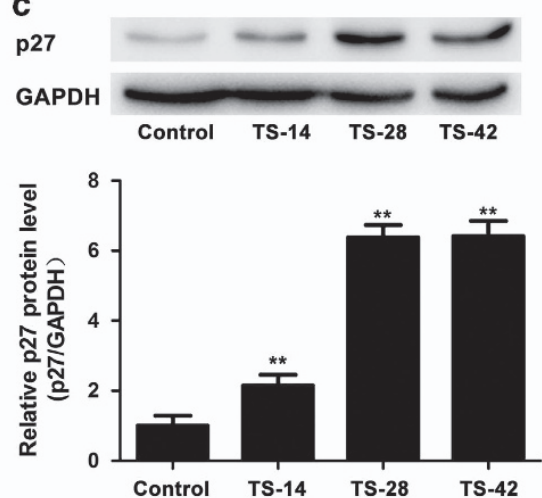

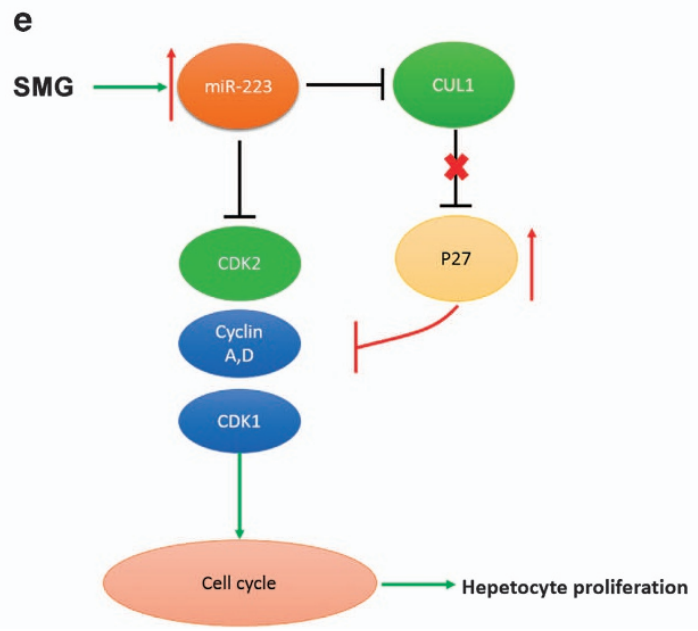

Figure 6 Upregulation of p27 expression in vitro and in vivo. (a) Immunofluorescence staining of p27 in the rat liver of the control, TS-14, TS-28 and TS-42 groups. Images were captured by a Leica TCS SP5 confocal scanning laser microscope. p27 in red (Cy3); nuclei in blue (DAPI). Scale bar, $50 \mu \mathrm{m}$. (b) Quantification of the number of p27-positive cells in the liver of the control, TS-14, TS-28 and TS-42 groups. $n=6$. (c) p27 protein expression level in the TS liver was significantly elevated compared with that of the control as shown by western blotting and semi-quantitative evaluation using Image J software. (d) The expression of the p27 protein in Hepa1-6 cells was examined $48 \mathrm{~h}$ after transfection with miR-233 mimic and negative control by western blotting and semi-quantitatively evaluated using Image J software. ${ }^{*} P<0.05,{ }^{* *} P<0.01$ compared to Control. (e) Diagram illustrating the regulatory hierarchy among miR-223 and its targets CDK2 and CUL1 in the control of hepatocyte proliferation.

was significantly elevated in the rat liver of the TS groups compared with that of control. Previous studies have demonstrated that miR-223 regulated the cell cycle by targeting E2F1, Fbxw7/Cdc4, IGF-1R, Cdk2 and TOX. ${ }^{37-40}$ Overexpression of miR-223 resulted in the downregulation of c-Myc in HepG2 cells and growth inhibition of $\mathrm{HBx}$-transfected HepG2 cells. ${ }^{54}$ Here we also observed that the proliferation of Hepal-6 cells was significantly decreased after miR-223 mimic transfection.
Our results and previous reports suggested that miR-223 may play a key role in the regulation of hepatocyte proliferation under SMG.

To address how miR-223 affected hepatocyte cell growth, some potential and putative mRNA targets were identified, such as CDK2 and CUL1. CDK2 has been shown to be a target of miR-223 in a Lewis lung carcinoma cell line. ${ }^{39}$ CDK2 is a member of the cyclin-dependent kinase family, which is 
essential for the G1/S transition and negatively regulated by p21 ${ }^{\mathrm{Cip} 1}$ (CDKN1A) and p27 ${ }^{\mathrm{Kip} 1}$ (CDKN1B). ${ }^{55}$ We confirmed that CDK2 was one of miR-223 targets by using a dual luciferase reporter system, and the expression level of CDK2 could be modulated by miR-223 in Hepal-6 cells in vitro. The upregulation of miR-223 was negatively associated with the decrease in CDK2 in the TS rat liver. Our data suggested that the elevated levels of miR-223 in hepatocytes could contribute to the decreased expression of the positive cell control factor CDK2, which helped to explain the reason for the dysregulated cell growth in the liver under SMG.

CUL1 is an essential rigid scaffold component of the SCF E3 ubiquitin ligase complex, which plays an important role in protein ubiquitination and degradation of p27 to control the cell cycle transition. ${ }^{48,56}$ It is well known that p27 is a universal cyclin-dependent kinase inhibitor, which binds several different classes of cyclin and Cdk molecules, such as cyclin D, Cdk4, Cdk1, cyclin E/Cdk2 and cyclin A/Cdk2 to control cell proliferation and tumor progression. ${ }^{57-59}$ Here we demonstrated that CUL1 was a direct target of miR-223 and that the p27 protein level was negatively correlated with CUL1 and positively associated with miR-223 in the TS rat liver and miR-223-transfected Hepa1-6 cells. Overexpression of CUL1 promoted cell proliferation via control of the cell cycle progression, which was facilitated by the degradation of $\mathrm{p} 27$ through the functional SCF ${ }^{\text {skp2 }}$ complex. ${ }^{60}$ c-Myc enhanced ubiquitin-dependent p27 proteolysis by directly activating expression of the CUL1 gene. ${ }^{61}$ Moreover, cyclin-CDK2 phosphorylated the threonine-187 on p27, which facilitated its interaction with SCF and resulted in degradation. ${ }^{62}$ The inhibition of CDK2 and CUL1 by miR-223 was associated with the elevation of p27 to inhibit hepatocyte proliferation in rats under SMG. The increased p27 protein levels were also consistent with the decreased mRNA level of CCNA2, CCND1 and $C D K 1$ in the rat liver under SMG. Interestingly, binding of p27 to mature miR-223 was shown to protect miR-223 from degradation in breast cancer. ${ }^{63}$ Our study, together with the results of previous studies, highlighted the complexity of miR-223 and p27 in cell cycle control. It is worth evaluating the changes of miR-223 and the rescue capability of miR-223 knockdown on hepatocyte proliferation in the liver during long-term spaceflight or SMG. Therefore, our findings suggest that miR-223/CDK2/CUL1/p27 signaling may play an important regulatory role in hepatocytes under SMG.

Collectively, miRNAs have been shown to have specific biological functions under microgravity. We demonstrated herein that elevated miR-223 in the rat liver was accompanied by inhibition of hepatocyte proliferation under SMG and discovered that a miR-223/CDK2/CUL1/p27 signaling pathway in the liver served as a pivotal anti-cell proliferation factor (Figure 6e). Our findings provide evidence for a new role for miR-223 in the regulation of the physiological and pathological function of the liver relevant to with clinical relevance and relevance to future spaceflight.

\section{CONFLICT OF INTEREST}

The authors declare no conflict of interest.

\section{ACKNOWLEDGEMENTS}

This work was supported in part by the National Natural Science Foundation (nos 31470832 and 31570858); National Instrumentation Program of China (nos 2012YQ0401400901 and 2013YQ190467); the State Key Laboratory of Space Medicine Fundamentals and Application, China Astronaut Research and Training Center (SMFA12B02, SYFD1500604); and the Advanced Space Medico-Engineering Research Project of China (2012SY54A1602, FTKY201305).

1 Longnecker DE, Worth MH Jr, Manning FJ. Review of NASA's Longitudinal Study of Astronaut Health. National Academies Press: Washington DC, NY, USA, 2004.

2 Honda Y, Honda S, Narici M, Szewczyk NJ. Spaceflight and ageing: reflecting on Caenorhabditis elegans in space. Gerontology 2014; 60: 138-142.

3 Merrill A, Wang $E$, Jones $D$, Hargrove J. Hepatic function in rats after spaceflight: effects on lipids, glycogen, and enzymes. Am J Physiol Regul Integr Comp Physiol 1987; 252: R222-R226.

4 Hollander J, Gore M, Fiebig R, Mazzeo R, Ohishi S, Ohno H et al. Spaceflight downregulates antioxidant defense systems in rat liver. Free Radic Biol Med 1998; 24: 385-390.

5 Merrill A, Hoel M, Wang E, Mullins R, Hargrove J, Jones D et al. Altered carbohydrate, lipid, and xenobiotic metabolism by liver from rats flown on Cosmos 1887. FASEB J 1990; 4: 95-100.

6 Hargrove J, Jones D. Hepatic enzyme adaptation in rats after space flight. Physiologist 1985; 28: S230.

7 Vetrova E, Drozdova T, Tigranian R, Shul'zhenko E. Energy-metabolism enzymes during combined exposure of the body to simulated weightlessness and gravitational overloads. Kosm Biol Aviakosm Med 1980; 15: 34-38.

8 Rivera CA, Tcharmtchi MH, Mendoza L, Smith CW. Endotoxemia and hepatic injury in a rodent model of hindlimb unloading. J App/ Physiol 2003; 95: 1656-1663.

9 Du F, Ding Y, Zou J, Li Z, Tian J, She R et al. Morphology and molecular mechanisms of hepatic injury in rats under simulated weightlessness and the protective effects of resistance training. PIOS ONE 2015; 10: e0127047.

10 Fausto N, Campbell JS, Riehle KJ. Liver regeneration. Hepatology 2006; 43: S45-S53.

11 Font-Burgada J, Shalapour S, Ramaswamy S, Hsueh B, Rossell D, Umemura A et al. Hybrid periportal hepatocytes regenerate the injured liver without giving rise to cancer. Cell 2015; 162: 766-779.

12 Palmes D, Spiegel HU. Animal models of liver regeneration. Biomaterials 2004; 25: 1601-1611.

13 Miyaoka Y, Miyajima A. To divide or not to divide: revisiting liver regeneration. Cell Div 2013; 8: 8.

14 Yamada Y, Kirillova I, Peschon JJ, Fausto N. Initiation of liver growth by tumor necrosis factor: deficient liver regeneration in mice lacking type I tumor necrosis factor receptor. Proc Natl Acad Sci USA 1997; 94: 1441-1446.

15 Cressman DE, Greenbaum LE, DeAngelis RA, Ciliberto G, Furth EE, Poli V et al. Liver failure and defective hepatocyte regeneration in interleukin-6deficient mice. Science 1996; 274: 1379-1383.

16 da Silva CG, Studer P, Skroch M, Mahiou J, Minussi DC, Peterson CR et al. A20 promotes liver regeneration by decreasing SOCS3 expression to enhance IL-6/STAT3 proliferative signals. Hepatology 2013; 57: 2014-2025.

17 Bei Y, Song Y, Wang F, Dimitrova-Shumkovska J, Xiang Y, Zhao Y et al. miR-382 targeting PTEN-Akt axis promotes liver regeneration. Oncotarget 2016; 7: 1584-1597.

18 Chen X, Song M, Chen W, Dimitrova-Shumkovska J, Zhao Y, Cao Y et al. MicroRNA-21 contributes to liver regeneration by targeting PTEN. Med Sci Monit 2016; 22: 83-91.

19 Tanimizu N, Kobayashi S, Ichinohe N, Mitaka T. Downregulation of miR122 by grainyhead-like 2 restricts the hepatocytic differentiation potential of adult liver progenitor cells. Development 2014; 141: 4448-4456.

20 John K, Hadem J, Krech T, Wahl K, Manns MP, Dooley S et al. MicroRNAs play a role in spontaneous recovery from acute liver failure. Hepatology 2014; 60: 1346-1355. 
21 Cheng Z, Ma R, Tan W, Zhang L. MiR-152 suppresses the proliferation and invasion of NSCLC cells by inhibiting FGF2. Exp Mol Med 2014; 46: e112.

$22 \mathrm{Ng}$ R, Song G, Roll GR, Frandsen NM, Willenbring H. A microRNA-21 surge facilitates rapid cyclin D1 translation and cell cycle progression in mouse liver regeneration. J Clin Invest 2012; 122: 1097-1108.

23 Yuan Q, Loya K, Rani B, Mobus S, Balakrishnan A, Lamle J et al. MicroRNA-221 overexpression accelerates hepatocyte proliferation during liver regeneration. Hepatology 2013; 57: 299-310.

24 Pan C, Chen H, Wang L, Yang S, Fu H, Zheng Y et al. Down-regulation of MiR-127 facilitates hepatocyte proliferation during rat liver regeneration. PLOS ONE 2012; 7: e39151.

25 Zhou J, Ju W, Wang D, Wu L, Zhu X, Guo Z et al. Down-regulation of microRNA-26a promotes mouse hepatocyte proliferation during liver regeneration. PLOS ONE 2012; 7: e33577.

26 Qi F, Hu JF, Liu BH, Wu CQ, Yu HY, Yao DK et al. MiR-9a-5p regulates proliferation and migration of hepatic stellate cells under pressure through inhibition of Sirt1. World J Gastroenterol 2015; 21: 9900-9915.

27 Chen Z, Ma T, Huang C, Zhang L, Lv X, Xu T et al. MiR-27a modulates the MDR1/P-glycoprotein expression by inhibiting FZD7/beta-catenin pathway in hepatocellular carcinoma cells. Cell Signal 2013; 25: 2693-2701.

28 Wang G, Zhu S, Gu Y, Chen Q, Liu X, MicroRNA-145 FuH. and MicroRNA-133a inhibited proliferation, migration, and invasion, while promoted apoptosis in hepatocellular carcinoma cells via targeting FSCN1. Dig Dis Sci 2015; 60: 3044-3052.

29 Hua HW, Jiang F, Huang Q, Liao Z, Ding G. MicroRNA-153 promotes Wnt/beta-catenin activation in hepatocellular carcinoma through suppression of WWOX. Oncotarget 2015; 6: 3840-3847.

30 Yu ZY, Bai YN, Luo LX, Wu H, Zeng Y. Expression of microRNA-150 targeting vascular endothelial growth factor-A is downregulated under hypoxia during liver regeneration. Mol Med Rep 2013; 8: 287-293.

31 Johnnidis JB, Harris MH, Wheeler RT, Stehling-Sun S, Lam MH, Kirak O et al. Regulation of progenitor cell proliferation and granulocyte function by microRNA-223. Nature 2008; 451: 1125-1129.

$32 \mathrm{Yu} \mathrm{CH}, \mathrm{Xu}$ CF, Li YM. Association of microRNA-223 expression with hepatic ischemia/reperfusion injury in mice. Dig Dis Sci 2009; 54: 2362-2366.

33 Vickers KC, Landstreet SR, Levin MG, Shoucri BM, Toth CL, Taylor RC et al. MicroRNA-223 coordinates cholesterol homeostasis. Proc Natl Acad Sci USA 2014; 111: 14518-14523.

34 Lu H, Buchan RJ, Cook SA. MicroRNA-223 regulates Glut4 expression and cardiomyocyte glucose metabolism. Cardiovasc Res 2010; 86: 410-420.

35 Laios A, O'Toole S, Flavin R, Martin C, Kelly L, Ring M et al. Potential role of miR-9 and miR-223 in recurrent ovarian cancer. Mol Cancer 2008; 7: 35.

36 Wei Y, Yang J, Yi L, Wang Y, Dong Z, Liu Z et al. MiR-223-3p targeting SEPT6 promotes the biological behavior of prostate cancer. Sci Rep 2014; 4: 7546.

37 Pulikkan JA, Dengler V, Peramangalam PS, Peer Zada AA, Muller-Tidow C, Bohlander SK et al. Cell-cycle regulator E2F1 and microRNA-223 comprise an autoregulatory negative feedback loop in acute myeloid leukemia. Blood 2010; 115: 1768-1778.

38 Xu Y, Sengupta T, Kukreja L, Minella AC. MicroRNA-223 regulates cyclin E activity by modulating expression of F-box and WD-40 domain protein 7 . J Biol Chem 2010; 285: 34439-34446.

39 Nian W, Ao X, Wu Y, Huang Y, Shao J, Wang Y et al. miR-223 functions as a potent tumor suppressor of the Lewis lung carcinoma cell line by targeting insulin-like growth factor-1 receptor and cyclin-dependent kinase 2. Oncol Lett 2013; 6: 359-366.

40 McGirt LY, Adams CM, Baerenwald DA, Zwerner JP, Zic JA, Eischen CM. miR-223 regulates cell growth and targets proto-oncogenes in mycosis fungoides/cutaneous T-cell lymphoma. J Invest Dermatol 2014; 134: 1101-1107.

41 Mangala LS, Zhang Y, He Z, Emami K, Ramesh GT, Story M et al. Effects of simulated microgravity on expression profile of microRNA in human lymphoblastoid cells. J Biol Chem 2011; 286: 32483-32490.

42 Zhang Y, Lu T, Wong M, Wang X, Stodieck L, Karouia F et al. Transient gene and microRNA expression profile changes of confluent human fibroblast cells in spaceflight. FASEB J 2016; 30: 2211-2224.

43 Wang $\mathrm{H}$, Sun Z, Wang Y, Hu Z, Zhou H, Zhang L et al. miR-33-5p, a novel mechano-sensitive microRNA promotes osteoblast differentiation by targeting Hmga2. Sci Rep 2016; 6: 23170.

44 Wang X, Guo B, Li Q, Peng J, Yang Z, Wang A et al. miR-214 targets ATF4 to inhibit bone formation. Nat Med 2013; 19: 93-100.
45 Wronski TJ, Morey-Holton ER. Skeletal response to simulated weightlessness: a comparison of suspension techniques. Aviat Space Environ Med 1987; 58: 63-68.

46 Jonscher KR, Alfonso-Garcia A, Suhalim JL, Orlicky DJ, Potma EO, Ferguson VL et al. Spaceflight activates lipotoxic pathways in mouse liver. PLOS ONE 2016; 11: e0152877.

47 Sheaff RJ, Groudine M, Gordon M, Roberts JM, Clurman BE. Cyclin E-CDK2 is a regulator of p27Kip1. Genes Dev 1997; 11: 1464-1478.

48 Wei W, Ayad NG, Wan Y, Zhang GJ, Kirschner MW, Kaelin WG Jr. Degradation of the SCF component Skp2 in cell-cycle phase G1 by the anaphase-promoting complex. Nature 2004; 428: 194-198.

49 Baek WY, Lee MA, Jung JW, Kim SY, Akiyama H, de Crombrugghe B et al. Positive regulation of adult bone formation by osteoblast-specific transcription factor osterix. J Bone Miner Res 2009; 24: 1055-1065.

50 Macho L, Nemeth S, Kvetnansky R, Fickova M, Tigranian RA, Serova L. Metabolic changes in the animals subjected to space flight. Acta Astronaut 1982; 9: 385-389.

51 Abraham S, Klein HP, Lin CY, Volkmann C. The effects of space flight on some rat liver enzymes regulating carbohydrate and lipid metabolism. Adv Space Res 1981; 1: 199-217.

52 Selbach M, Schwanhausser B, Thierfelder N, Fang Z, Khanin R, Rajewsky $\mathrm{N}$. Widespread changes in protein synthesis induced by microRNAs. Nature 2008; 455: 58-63.

53 Guo H, Ingolia NT, Weissman JS, Bartel DP. Mammalian microRNAs predominantly act to decrease target mRNA levels. Nature 2010; 466: 835-840.

54 Yu G, Chen X, Chen S, Ye W, Hou K, Liang M. MiR-19a, miR-122 and miR-223 are differentially regulated by hepatitis $B$ virus $X$ protein and involve in cell proliferation in hepatoma cells. J Trans/ Med 2016; 14: 122.

55 Levkau B, Koyama H, Raines EW, Clurman BE, Herren B, Orth K et al. Cleavage of p21Cip1/Waf1 and p27Kip1 mediates apoptosis in endothelial cells through activation of Cdk2: role of a caspase cascade. Mol Cell 1998; 1: 553-563.

56 Morimoto M, Nishida T, Honda R, Yasuda H. Modification of cullin-1 by ubiquitin-like protein Nedd8 enhances the activity of SCF(skp2) toward p27(kip1). Biochem Biophys Res Commun 2000; 270: 1093-1096.

57 Toyoshima H, Hunter T. p27, a novel inhibitor of G1 cyclin-Cdk protein kinase activity, is related to p21. Cell 1994; 78: 67-74.

58 Polyak K, Lee MH, Erdjument-Bromage H, Koff A, Roberts JM, Tempst P et al. Cloning of p27Kip1, a cyclin-dependent kinase inhibitor and a potential mediator of extracellular antimitogenic signals. Cell 1994; 78: 59-66.

59 Kotoshiba S, Gopinathan L, Pfeiffenberger E, Rahim A, Vardy LA, Nakayama $\mathrm{K}$ et al. p27 is regulated independently of Skp2 in the absence of Cdk2. Biochim Biophys Acta 2014; 1843: 436-445.

60 Chen G, Li G. Increased Cul1 expression promotes melanoma cell proliferation through regulating p27 expression. Int J Oncol 2010; 37: 1339-1344.

61 O'Hagan RC, Ohh M, David G, de Alboran IM, Alt FW, Kaelin WG Jr et al. Myc-enhanced expression of Cul 1 promotes ubiquitin-dependent proteolysis and cell cycle progression. Genes Dev 2000; 14: 2185-2191.

62 Montagnoli A, Fiore F, Eytan E, Carrano AC, Draetta GF, Hershko A et al. Ubiquitination of p27 is regulated by Cdk-dependent phosphorylation and trimeric complex formation. Genes Dev 1999; 13: 1181-1189.

63 Armenia J, Fabris L, Lovat F, Berton S, Segatto I, D'Andrea S et al. Contact inhibition modulates intracellular levels of miR-223 in a p27kip1dependent manner. Oncotarget 2014; 5: 1185-1197.

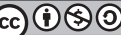

This work is licensed under a Creative Commons Attribution-NonCommercial-ShareAlike 4.0 International License. The images or other third party material in this article are included in the article's Creative Commons license, unless indicated otherwise in the credit line; if the material is not included under the Creative Commons license, users will need to obtain permission from the license holder to reproduce the material. To view a copy of this license, visit http:// creativecommons.org/licenses/by-nc-sa/4.0/

Supplementary Information accompanies the paper on Experimental \& Molecular Medicine website (http://www.nature.com/emm) 